\title{
Effect of gestational diabetes mellitus on newborn cholesterol metabolism
}

\section{Miettinen, Helena E.}

2018-08

Miettinen , H E , Rono , K , Koivusalo , S B , Eriksson, J G \& Gylling , H 2018 , ' Effect of gestational diabetes mellitus on newborn cholesterol metabolism ' , Atherosclerosis, vol. 275 , pp. 346-351 . https://doi.org/10.1016/j.atherosclerosis.2018.06.879

http://hdl.handle.net/10138/245182

https://doi.org/10.1016/j.atherosclerosis.2018.06.879

unspecified

publishedVersion

Downloaded from Helda, University of Helsinki institutional repository.

This is an electronic reprint of the original article.

This reprint may differ from the original in pagination and typographic detail.

Please cite the original version. 


\title{
Effect of gestational diabetes mellitus on newborn cholesterol metabolism
}

\author{
Helena E. Miettinen ${ }^{\text {a, }}{ }^{\text {, }}$, Kristiina Rönö ${ }^{\text {b }}$, Saila B. Koivusalo ${ }^{\text {b }}$, Johan G. Eriksson ${ }^{\text {c, d }}$, \\ Helena Gylling ${ }^{\mathrm{e}}$ \\ ${ }^{a}$ University of Helsinki and Helsinki University Hospital, Endocrinology, Finland \\ ${ }^{\mathrm{b}}$ University of Helsinki and Helsinki University Hospital, Obstetrics and Gynaecology, Women's Hospital, P.O. Box 140 Haartmaninkatu 2, 00029 HUS, \\ Finland \\ ${ }^{\mathrm{c}}$ University of Helsinki and Helsinki University Hospital, General Practice and Primary Health Care, Folkhälsan Research Center, P.O. Box 20 \\ (Tukholmankatu 8 B, 6. Fl), Biomedicum, Helsinki, 00014, Finland \\ ${ }^{\mathrm{d}}$ University of Helsinki National Institute for Health and Welfare, Chronic Disease Prevention, Finland \\ e University of Helsinki and Helsinki University Hospital, Internal Medicine, P.O. BOX 700, 00029 HUS, Helsinki, Finland
}

\section{A R T I C L E I N F O}

\section{Article history:}

Received 31 March 2018

Received in revised form

20 June 2018

Accepted 21 June 2018

Available online 22 June 2018

\section{Keywords:}

Pregnancy

Phytosterol

Cholesterol metabolism

Maternal-fetal cholesterol transport

Lipoprotein

Gestational diabetes mellitus

\begin{abstract}
A B S T R A C T
Background and aims: Impaired glucose metabolism during pregnancy may associate with changes in fetal cholesterol metabolism. We investigated if gestational diabetes mellitus (GDM) affects newborn cholesterol metabolism as determined by cord blood squalene and non-cholesterol sterols. Furthermore, we examined potential correlations between cord blood and maternal serum non-cholesterol sterols. Methods: Pregnant women at risk for GDM $\left(\mathrm{BMI}>30 \mathrm{~kg} / \mathrm{m}^{2}\right)$ were enrolled from maternity clinics in Finland. GDM was determined from the results of an oral glucose tolerance test. Serum samples were taken in the third trimester of pregnancy, and cord blood samples collected from their newborns at birth. Squalene and non-cholesterol sterols were analyzed from serum and cord blood by gas liquid chromatography. All women with GDM were in good glycaemic control.

Results: The ratios of squalene and non-cholesterol sterols to cholesterol $(100 \times \mu \mathrm{mol} / \mathrm{mmol}$ of cholesterol) in cord blood did not differ between the infants born to mothers with GDM $(n=15)$ or mothers with normal glucose tolerance $(\mathrm{n}=13)$. The ratios of sitosterol and campesterol to cholesterol in the cord blood correlated with the corresponding maternal serum ratios $(r=0.70, p<0.0001)$ in both groups. Conclusions: In obese women under good glycaemic control, GDM did not affect newborn cholesterol metabolism. Cord blood sitosterol and campesterol ratios to cholesterol correlated with the corresponding maternal serum ratios thus potentially reflecting maternal-fetal cholesterol transport.
\end{abstract}

(C) 2018 Elsevier B.V. All rights reserved.

\section{Introduction}

Incidence of obesity and gestational diabetes (GDM) are increasing in western countries, leading to a growing number of GDM associated adverse pregnancy outcomes [1]. These GDM related risks, most importantly fetal macrosomia, are mediated at least partly via disturbed glucose- and/or lipid metabolism. Maternal plasma lipids might be stronger determinants for fetal

$$
\text { test. }
$$

Abbreviations: GDM, gestational diabetes mellitus; OGTT, oral glucose tolerance

* Corresponding author. Department of Endocrinology, Helsinki University Hospital, Biomedicum Helsinki C422, PL 700, 00029 HUS, Finland.

E-mail address: helena.miettinen@hus.fi (H.E. Miettinen). growth than plasma glucose levels [2], reviewed in Ref. [3]. Studies focusing upon serum lipids and lipoproteins in GDM have been controversial showing increase, decrease or no change in serum triglyceride, total- or LDL cholesterol levels [4-7], reviewed in Ref. [3].

In addition to cholesterol, serum contains cholesterol precursors (squalene, lanosterol, cholestenol, desmosterol and lathosterol), which reflect cholesterol synthesis, and cholestanol, a derivative of cholesterol, and plant sterols (campesterol, sitosterol and avenasterol), which reflect cholesterol absorption efficiency in many conditions [8]. We have previously shown that cholesterol synthesis markers in obese GDM pregnancies were increased when compared to obese control pregnancies with normal glucose tolerance. Furthermore, in GDM, the second trimester maternal 
serum squalene concentration correlated with neonatal birth weight suggesting potential contribution of maternal cholesterol metabolism to fetal weight [7].

Although several studies have investigated maternal serum and cord blood lipids in GDM, less is known about the impact of GDM on placental and fetal cholesterol metabolism. In some studies, infants of diabetic mothers have had higher plasma cholesterol and phospholipid levels, or altered HDL composition whereas others failed to show any differences [9-11]. Impaired glucose metabolism during pregnancy could associate with changes in fetal cholesterol metabolism, detectable by analysing cord blood squalene and noncholesterol sterols. To our knowledge, no studies have explored cholesterol metabolism of newborns of GDM mothers. Nor is there data available about potential associations of maternal noncholesterol sterols in GDM with those in cord blood.

Thus, the present study was undertaken to examine serum cholesterol precursors, cholestanol, and plant sterols in cord blood of infants born to mothers with GDM or normal glucose tolerance. Furthermore, we evaluated potential correlations between fetal and maternal non-cholesterol sterols.

\section{Materials and methods}

\subsection{Subjects}

All pregnant study subjects were participants of our previous study [7] and originated from the control arm of the Finnish RADIEL-study [12]. Characteristics of the pregnant study subjects, as well as their serum non-cholesterol sterol concentrations and ratios to cholesterol, have been described previously in detail [7]. Briefly, pregnant women classified as at risk for developing GDM $\left(\mathrm{BMI}>30 \mathrm{~kg} / \mathrm{m}^{2}\right)$ were enrolled to the study at the time of their first visit to the maternity clinics in Southern Finland. Exclusion criteria for all subjects included previous diabetes, undiagnosed thyroid disease (abnormal serum thyroid stimulating hormone (TSH) concentration), use of insulin before samples were taken, age $<18$ years, oral corticosteroid treatment, alcohol or drug abuse, multiple pregnancy, physical disability, significant co-operation difficulties (e.g. insufficient language skills) or history of severe psychiatric disorder or hypertension. An oral $75 \mathrm{~g}$ 2-hr glucose tolerance test (OGTT) was performed to all subjects at weeks 22-29 of pregnancy, with the exception of 3 subjects with OGTT performed at weeks 31-33. OGTT was considered diagnostic for GDM if any of the measures were pathological. The following diagnostic thresholds were used: fasting plasma glucose $>5.3 \mathrm{mmol} / \mathrm{L}, 1 \mathrm{~h}$ plasma glucose $\geq 10.0 \mathrm{mmol} / \mathrm{L}$ or $2 \mathrm{~h}$ plasma glucose $\geq 8.6 \mathrm{mmol} / \mathrm{L}$ [13]. With the exception of two subjects all study subjects were of Finnish origin.

Cord blood samples were collected when technically feasible from all subjects $(n=28)$ participating in our previous study [7]. The GDM group $(n=15)$ consisted of women having a pathological OGTT while the control subjects $(n=13)$ had a normal OGTT during pregnancy and no history of previous GDM.

The newborns were weighed directly after delivery on a digital baby scale. The relative weight was calculated using Finnish standards at the time of the study adjusted for sex and gestational age [14].

\subsection{Methods}

Serum samples were collected from all subjects during pregnancy weeks 34-36 as described previously [12]. Cord blood samples were collected at birth and stored at $-80^{\circ} \mathrm{C}$ until analyzed. Serum and cord blood cholesterol and cholesterol precursors (squalene, lanosterol, dihydrolanosterol, $\Delta^{8}$ dimethylsterol, $\Delta^{8}$ methostenol, $\Delta^{7}$ methostenol, cholestenol, desmosterol and lathosterol), cholestanol and plant sterols (campesterol, sitosterol and avenasterol) were assayed by gas liquid chromatography as described [15]. Shortly, serum/cord blood squalene and noncholesterol sterols were quantified from non-saponifiable materials by capillary gas liquid chromatography (Agilent $6890 \mathrm{~N}$ Network GC System, Agilent Technologies, Wilmington, DE) equipped with a $50 \mathrm{~m}$ long non-polar Ultra 2 capillary column (5\% phenyl-methyl siloxane; Agilent Technologies, Wilmington, DE) with $5 \alpha$-cholestane as internal standard [15]. The serum and cord blood values of squalene and non-cholesterol sterols are expressed as concentrations $(\mu \mathrm{g} / \mathrm{dL})$ or ratios to cholesterol $(100 \times \mu \mathrm{mol} /$ mmol of cholesterol) by dividing squalene and non-cholesterol sterol concentrations with the cholesterol value of the same gas liquid chromatography run in order to eliminate the effect of different cholesterol levels [16].

Serum and cord blood triglycerides, total-, LDL-, and HDL cholesterol concentrations were determined by enzymatic methods, Thermo Konelab GO (Thermo Scientific, Vantaa, Finland).

\subsection{Statistical analyses}

Statistical analyses were performed with IBM SPSS 22.0 statistics program. Normality was tested before further analyses. Student's t-test or Mann-Whitney $U$ test were used to analyze differences in between the groups. Spearman's correlation was used to analyze correlation between parameters. $p$-value $<0.05$ was considered statistically significant.

\subsection{Ethical approval}

The study was performed according to the principles of the Declaration of Helsinki and was approved by the Ethical Committees of the Helsinki University Central Hospital (Dnro 300/E9/06) and South Karelia Central Hospital (Dnro M06/08). Trial Registration: Clinicaltrials.gov Identifier: NCT01698385. All participants signed an informed consent form.

\section{Results}

The GDM and control groups did not differ in age, nor were there any differences in serum total-, HDL-, LDL cholesterol, triglyceride or insulin levels. Subjects with GDM had higher BMI and plasma glucose concentration but they were in good glycaemic control (Table 1).

Characteristics of the infants born to mothers with GDM (GDM group, $\mathrm{n}=15$ ) or mothers with normal glucose tolerance (control group, $\mathrm{n}=13$ ) are shown in Table 2 . The GDM and control groups,

Table 1

Characteristics of women with GDM and control subjects, and their serum total and lipoprotein cholesterol, triglyceride, glucose and insulin concentrations in the third trimester of pregnancy.

\begin{tabular}{lll}
\hline & $\begin{array}{l}\text { Control } \\
\mathrm{n}=13\end{array}$ & $\begin{array}{l}\text { GDM } \\
\mathrm{n}=15\end{array}$ \\
\hline Age (years) & $29.9 \pm 1.9$ & $31.1 \pm 0.9$ \\
BMI $\left(\mathrm{kg} / \mathrm{m}^{2}\right)$ & $35.5 \pm 0.8$ & $39.2 \pm 1.2^{a}$ \\
Glucose $(\mathrm{mmol} / \mathrm{L})$ & $4.59 \pm 0.08$ & $5.11 \pm 0.13^{b}$ \\
Insulin $(\mathrm{mU} / \mathrm{L})$ & $13.99 \pm 2.02$ & $15.88 \pm 2.24$ \\
Total cholesterol $(\mathrm{mmol} / \mathrm{L})$ & $6.00 \pm 0.22$ & $5.80 \pm 0.18$ \\
HDL cholesterol $(\mathrm{mmol} / \mathrm{L})$ & $1.73 \pm 0.10$ & $1.63 \pm 0.14$ \\
LDL cholesterol $(\mathrm{mmol} / \mathrm{L})$ & $3.45 \pm 0.26$ & $3.10 \pm 0.21$ \\
Triglycerides $(\mathrm{mmol} / \mathrm{L})$ & $2.35 \pm 0.21$ & $2.53 \pm 0.18$ \\
\hline a $p<0.05$. & & \\
${ }^{\mathrm{b}} p<0.01$. & & \\
Values are mean $\pm \mathrm{SE}$. & &
\end{tabular}


Table 2

Characteristics of the infants born to control mothers and mothers affected by GDM and their cord blood total and lipoprotein cholesterol and triglyceride concentrations.

\begin{tabular}{lll}
\hline & Control $\mathrm{n}=13$ & GDM $\mathrm{n}=15$ \\
\hline Gestational age (weeks) & $40.54 .7 \pm 0.32$ & $39.8 \pm 0.33$ \\
Birth weight $(\mathrm{kg})$ & $3.54 \pm 0.13$ & $3.50 \pm 0.12$ \\
Relative weight & $-0.25 \pm 0.27$ & $-0.09 \pm 0.24$ \\
Boys/girls & $8 / 5$ & $5 / 10$ \\
Total cholesterol (mmol/L) & $1.55 \pm 0.11$ & $1.55 \pm 0.10$ \\
HDL cholesterol (mmol/L) & $0.55 \pm 0.05$ & $0.57 \pm 0.06$ \\
LDL cholesterol (mmol/L) & $0.79 \pm 0.09$ & $0.78 \pm 0.08$ \\
Total triglycerides $(\mathrm{mmol} / \mathrm{L})$ & $0.47 \pm 0.06$ & $0.44 \pm 0.05$ \\
\hline
\end{tabular}

Values are mean $\pm \mathrm{SE}$.

or boys and girls, did not differ in gestational age, weight or relative weight. Nor were there any differences in their cord blood total-, HDL-, LDL cholesterol or total triglyceride levels.

Cord blood concentrations of squalene and non-cholesterol sterols (data not shown), as well as their ratios to cholesterol, were similar in GDM and control groups (Table 3). In maternal serum dihydrolanosterol, $\Delta^{8}$ dimethylsterol, $\Delta^{8}$ methostenol and $\Delta^{7}$ methostenol were undetectable but in the cord blood they were present in high amounts, especially $\Delta^{8}$ methostenol and $\Delta^{7}$ methostenol. As cord blood total cholesterol concentration was low, the ratios of squalene, lanosterol, cholestenol, desmosterol, lathosterol and cholestanol were about 2-4 fold higher than the corresponding ratios in the maternal serum. Only the ratios of sitosterol and campesterol to cholesterol were lower in the cord blood than in maternal serum $\left(p<10^{-8}\right)$. In the maternal serum the major plant sterol was campesterol, and the ratio of campesterol to sitosterol was $1.9 \pm 0.05$, whereas in cord blood the ratio of campesterol to sitosterol was only $0.7 \pm 0.04$.

The ratio of cord blood sitosterol to cholesterol was $50.2 \% \pm 3.7$ and $56.8 \% \pm 3.0$ of the maternal serum ratios (control vs. GDM, respectively, mean $\pm \mathrm{SE}$ ), and varied between $30 \%$ and $85 \%$, while the corresponding percentages for campesterol were only $22.4 \pm 2.3 \%$ and $28.7 \pm 0.9 \%$ (control vs. GDM). The cord blood sitosterol concentration was $13.5 \pm 1.9 \%$ and $15.1 \pm 1.2 \%$ of the maternal serum concentrations (control vs. GDM, respectively,

Table 3

Total cholesterol $(\mathrm{mmol} / \mathrm{L})$ and squalene and non-cholesterol sterol ratios to cholesterol $(100 \times \mu \mathrm{mol} / \mathrm{mmol}$ of cholesterol $)$ in cord blood of infants born to mothers with GDM and in controls, and in the serum of their mothers in the third trimester of their pregnancy.

\begin{tabular}{llllll}
\hline & \multicolumn{2}{l}{ Cord blood } & & \multicolumn{2}{l}{ Maternal serum } \\
\cline { 2 - 3 } \cline { 5 - 6 } \cline { 5 - 6 } & Control & GDM & & Control & GDM \\
& $\mathrm{n}=13$ & $\mathrm{n}=15$ & $\mathrm{n}=12$ & $\mathrm{n}=15$ \\
\hline Total cholesterol & $1.5 \pm 0.1$ & $1.5 \pm 0.1$ & $6.0 \pm 0.2^{c}$ & $5.8 \pm 0.2^{c}$ \\
Squalene & $43.0 \pm 7.9$ & $31.3 \pm 4.3$ & $9.2 \pm 0.8^{a}$ & $11.3 \pm 0.8^{b}$ \\
Lanosterol & $34.4 \pm 6.1$ & $30.1 \pm 1.7$ & & $11.9 \pm 1.1^{a}$ & $12.8 \pm 1.1^{c}$ \\
Dihydrolanosterol & $25.2 \pm 2.0$ & $27.2 \pm 3.0$ & $\mathrm{ND}$ & $\mathrm{ND}$ \\
$\Delta^{8}$ Dimethylsterol & $41.5 \pm 8.1$ & $32.7 \pm 5.1$ & $\mathrm{ND}$ & $\mathrm{ND}$ \\
$\Delta^{8}$ Methostenol & $159.2 \pm 22.7$ & $156.5 \pm 15.8$ & $\mathrm{ND}$ & $\mathrm{ND}$ \\
$\Delta^{7}$ Methostenol & $136.8 \pm 29.3$ & $119.2 \pm 17.7$ & $\mathrm{ND}$ & $\mathrm{ND}$ \\
Cholestenol & $72.1 \pm 5.2$ & $72.3 \pm 5.4$ & & $17.4 \pm 1.4^{c}$ & $15.1 \pm 0.7^{c}$ \\
Desmosterol & $168.5 \pm 7.6$ & $152.8 \pm 5.3$ & $91.1 \pm 5.1^{c}$ & $84.4 \pm 3.7^{c}$ \\
Lathosterol & $319.6 \pm 21.8$ & $308.9 \pm 19.5$ & $142.9 \pm 14.0^{c}$ & $134.6 \pm 8.4^{c}$ \\
Cholestanol & $695.1 \pm 35.8$ & $694.7 \pm 19.6$ & $261.3 \pm 13.4^{c}$ & $253.6 \pm 9.3^{c}$ \\
Campesterol & $37.3 \pm 4.4$ & $29.9 \pm 2.1$ & & $179.2 \pm 20.0^{c}$ & $162.7 \pm 12.3^{c}$ \\
Sitosterol & $46.3 \pm 2.3$ & $46.0 \pm 1.8$ & $97.3 \pm 8.2^{c}$ & $84.5 \pm 5.8^{c}$ \\
\hline
\end{tabular}

$\mathrm{ND}=$ not detectable.

${ }^{\mathrm{a}} p<0.01$.

${ }^{\mathrm{b}} p<0.001$.

${ }^{c} p<0.0001$.

Values are mean $\pm \mathrm{SE}$; $p$-values are for cord blood $v s$. maternal serum. mean $\pm S E$ ), and again for campesterol the respective percentages were lower $(6.1 \pm 0.9 \%$ and $5.0 \pm 0.4 \%)\left(p<10^{-6}\right.$ sitosterol vs. campesterol).

Cord blood non-cholesterol sterols did not correlate with maternal BMI. We next looked at potential correlations between cord blood and third trimester maternal serum lipids and sterols. There were no correlations between maternal serum triglyceride, or total-, LDL- or HDL cholesterol concentrations and those in the cord blood. Neither were there any correlations between cholesterol precursor sterols in cord blood and in maternal serum. The high cholestanol concentration observed in both the cord blood and in maternal serum in the third trimester did not correlate with each other. However, the ratio of sitosterol to cholesterol in the cord blood correlated to the ratios of sitosterol $(r=0.70, p<0.0001)$ (Fig. 1), campesterol ( $\mathrm{r}=0.68, p<0.0001)$ and cholestanol $(\mathrm{r}=0.71$, $p<0.0001)$ in the maternal serum in both groups ( $p$-values for combined groups). Also the ratio of campesterol to cholesterol in the cord blood correlated with the maternal serum ratio of campesterol $(r=0.69, p<0.0001)$.

In the cord blood, total cholesterol concentration correlated with sitosterol $(r=0.78, p<0.00001)$, campesterol $(r=0.610$, $p=0.001)$, desmosterol $(\mathrm{r}=0.646, p<0.001)$ and cholestanol $(\mathrm{r}=0.67, p<0.0001)$ concentrations in both groups (Fig. 2). In addition, cholestanol and desmosterol concentrations correlated with sitosterol $(r=0.62, p<0.001$, and $r=0.44, p<0.05$, respectively) and campesterol ( $\mathrm{r}=0.47, p<0.05$ and $\mathrm{r}=0.47, p<0.05$ ) concentrations.

Birth weight or relative birth weight did not correlate with cord blood triglyceride, or total-, LDL- or HDL cholesterol concentration nor with squalene or non-cholesterol sterol concentrations or ratios to cholesterol. Cord blood triglyceride concentration correlated with the length of gestation in both GDM $(r=0.652, p=0.008)$ and control groups $(r=0.783, p=0.003)$.

\section{Discussion}

The present study explores for the first time cord blood squalene and non-cholesterol sterols in newborns from pregnancies affected

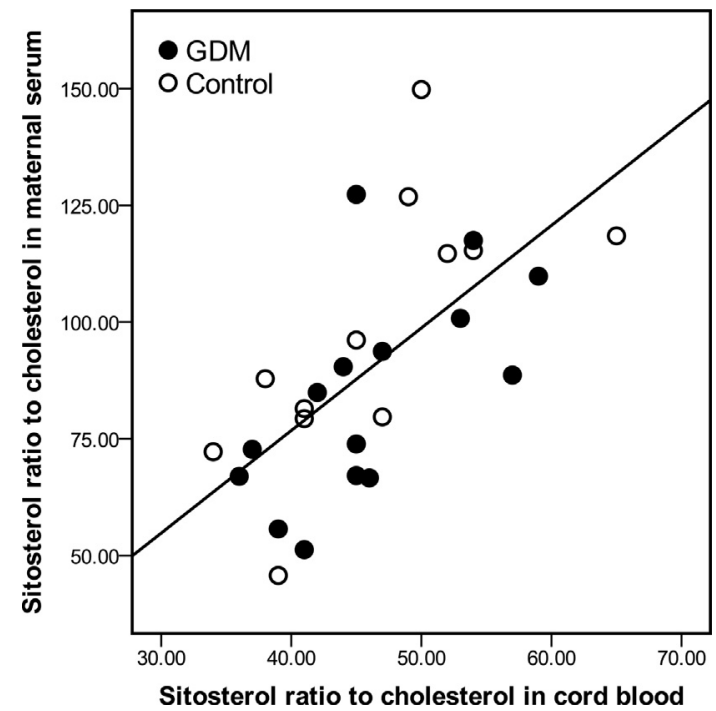

Fig. 1. Correlation between the cord blood ratios of sitosterol to cholesterol (100 $\times$ $\mu \mathrm{mol} / \mathrm{mmol}$ of cholesterol) and the third trimester maternal serum ratios of sitosterol to cholesterol $(100 \times \mu \mathrm{mol} / \mathrm{mmol}$ of cholesterol).

Open circle, control pregnancies; black circle, pregnancies affected by GDM $(r=0.70$, $p<0.0001$ ). 

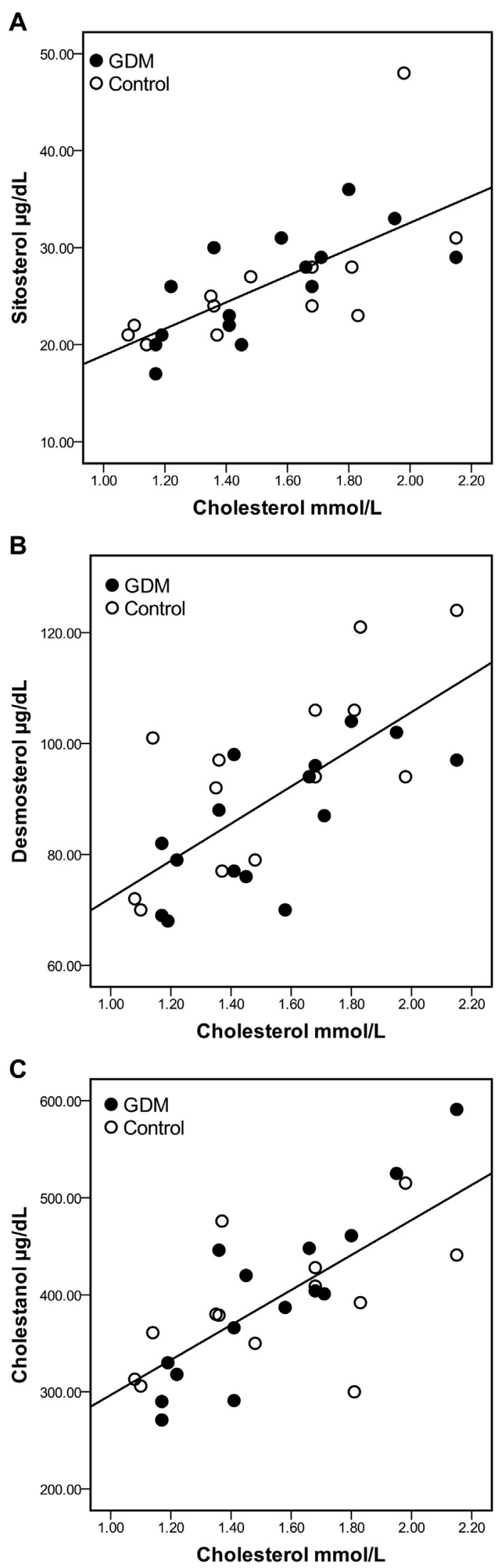

Fig. 2. Correlation between the cord blood cholesterol and (A) sitosterol $(r=0.78$, $p<0.00001)$, (B) desmosterol $(r=0.646, p<0.001)$ and (C) cholestanol $(r=0.67$, $\mathrm{p}<0.0001$ ) concentrations in control newborns (open circle) and newborns of mothers with GDM (filled circle). by obesity and GDM, and further investigates correlations between cord blood and maternal serum sterols. We proposed that impaired glucose metabolism during pregnancy could associate with changes in fetal cholesterol metabolism, detectable by analysing cord blood squalene and non-cholesterol sterols. The main findings in our study were that 1 . in obesity, GDM under good glycaemic control did not affect squalene or non-cholesterol sterol concentrations or ratios to cholesterol in cord blood. 2. The ratio of cord blood plant sterols correlated with the respective maternal serum plant sterol ratios potentially reflecting maternal-fetal cholesterol transport.

In all newborns, the presence of methylsterols and 2-4 fold higher ratios of lanosterol, desmosterol, lathosterol, cholestenol and squalene to cholesterol in the cord blood, as compared to corresponding maternal serum ratios, suggest that all steps of cholesterol synthesis were increased at birth, a finding similar to previous studies $[17,18]$.

The finding that total cholesterol concentration correlated with desmosterol, but not with lathosterol, concentration in cord blood suggests that the cholesterol synthesis pathway through the desmosterol i.e. Bloch route might have been preferred over the Kandutsch-Russell route at this point. It is apparently not explained by the effect of maternal obesity as similar correlation was also found in healthy control newborns (Vuorio AF, Miettinen HE and Gylling $\mathrm{H}$, unpublished data). It may be that, as desmosterol is highly abundant in the brain, its production may be important at the time of vigorous growth and development [19,20]. Further studies are required to examine the specific role, if any, of desmosterol in the newborns.

High levels of cholestanol found in cord blood, and the lack of its correlation to maternal serum cholestanol levels, may reflect either increased fetal cholestanol synthesis from cholesterol, supported by the finding that cholesterol concentration correlated with that of cholestanol, or decreased elimination via biliary excretion, i.e. mild cholestasis, due to immature biliary secretion in the newborns. Previously it has been shown that cholestanol levels drastically decrease by the first year of life, probably indicating development of biliary system, and/or decrease in cholesterol synthesis activity [18].

GDM in obese subjects did not have any significant impact on cord blood cholesterol or triglyceride concentrations, or newborn cholesterol metabolism as determined by cord blood squalene and non-cholesterol sterols. However, as all of our GDM subjects were in good glycaemic control, the potential impact of glycaemic variation on fetal cholesterol metabolism might have been abolished.

The lack of correlation between maternal and fetal cholesterol and triglyceride concentrations is in accordance with the previous studies [21]. Maternal and fetal cholesterol and lipoprotein levels do not commonly correlate in full term newborns [21,22] reviewed in Ref. [23], whereas in early stage of pregnancy, when cord blood cholesterol is higher, correlation may exist because at that time the fetus assumingly relies mainly on maternal cholesterol sources $[24,25]$. To our knowledge, associations of non-cholesterol sterols in cord blood with maternal serum sterols in GDM have not been examined before. At birth, the concentrations of cholesterol precursor sterols in cord blood are affected not only by the maternalfetal transport, but also by highly active fetal endogenous cholesterol synthesis. Thus, it is not surprising that concentrations, or ratios of cholesterol precursors in the cord blood did not associate with the corresponding maternal serum levels. As the samples from the mother were not taken simultaneously with the cord blood, this might have influenced the results [7], but in a previous and only study exploring simultaneous correlations of maternal and cord blood sterols at birth, no such correlations were detected either [17]. In that respect, the finding that the ratios of sitosterol and 
campesterol to cholesterol correlated with the corresponding maternal ratios, is of interest, and a new observation in GDM pregnancies [17]. Although a low number of the GDM subjects is a limitation to our study, a similar correlation was previously observed in healthy pregnancies [17].

As plant sterols cannot be synthesized, all sitosterol and campesterol present in the cord blood must originate from the maternal circulation and may thus reflect maternal-fetal cholesterol transport. Also, despite of increased cholesterol synthesis of the newborns, cord blood cholesterol concentration correlated with sitosterol and campesterol concentrations. Previously it was estimated that in cord blood of healthy fetuses, sitosterol ratios were $40-50 \%$ of the non-related pregnant levels [18]. In our study, percentages of sitosterol ratios in cord blood varied from 30 to $85 \%$ (average 54\%) of the corresponding maternal ratios, but were not affected by GDM or maternal BMI. On the other hand, the percentage of plant sterol concentrations in cord blood were only $15 \%$ of the maternal concentrations. Which of the parameters then, if any, reflects the actual extent of maternal-fetal cholesterol transport, requires further studies.

The amount of campesterol in serum is normally higher than that of sitosterol due to better absorption of campesterol from the intestine [26]. This was also seen in maternal serum, where the concentration of campesterol was almost twice as high as that of sitosterol. In this respect, it is interesting that in cord blood the ratio of campesterol to sitosterol was only 0.7. Plant sterols in serum are carried mainly in LDL particles, in which the campesterol to sitosterol ratio is similar to that of serum. The exact mechanisms and regulation of maternal-fetal cholesterol delivery are not yet completely understood. Maternal lipoproteins must first be taken up in the apical side of the trophoblasts, which express several lipoprotein receptors, reviewed in Ref. [27]. Excretion of cholesterol from the basolateral membrane to the fetal side may involve ABCA1 $[28,29]$, which is also capable of secreting plant sterols. Thus, at some point campesterol apparently is handled differently than sitosterol, as reflected by lower ratios of cord blood campesterol to maternal serum campesterol.

Further studies are required to find out if sitosterol and campesterol concentrations or ratios to cholesterol in the cord blood, or during pregnancy from amniotic fluid, could be used as a tool to determine activity of maternal-fetal cholesterol transport.

\subsection{Conclusion}

We have shown that, in obesity, and under good glycemic control, GDM did not have any effect on newborn cholesterol metabolism as determined by cord blood squalene and noncholesterol sterol concentrations or ratios to cholesterol. The ratios of sitosterol and campesterol to cholesterol in cord blood correlated with corresponding maternal serum ratios, apparently reflecting maternal-fetal cholesterol transport.

\section{Conflicts of interest}

The authors declared they do not have anything to disclose regarding conflict of interest with respect to this manuscript.

\section{Financial support}

This study was supported by a grant of Finnish Medical Foundation. The funding source had no involvement in study design; in the collection, analysis and interpretation of data; in the writing of the report; or in the decision to submit the article for publication.

\section{Author contributions}

HM has planned and executed the current study, analyzed the data and written the manuscript, KR participated in the planning and implementation of the study, prepared the database for statistical analyses, and participated in the editing the manuscript, SBK participated in the design of the study, and helped in editing of the paper, JGE has participated in planning of the study as well as critical revision of the manuscript and HG has planned and executed the current study and participated in writing and editing the manuscript.

\section{Acknowledgements}

We thank Ms Leena Kaipiainen and Ms Maaret Ahola for their excellent technical assistance.

\section{References}

[1] B.E. Metzger, L.P. Lowe, A.R. Dyer, E.R. Trimble, U. Chaovarindr, D.R. Coustan, et al., Hyperglycemia and adverse pregnancy outcomes, N. Engl. J. Med. 358 (19) (2008) 1991-2002.

[2] U.M. Schaefer-Graf, K. Graf, I. Kulbacka, S.L. Kjos, J. Dudenhausen, K. Vetter, et al., Maternal lipids as strong determinants of fetal environment and growth in pregnancies with gestational diabetes mellitus, Diabetes Care 31 (9) (2008) $1858-1863$.

[3] E. Herrera, H. Ortega-Senovilla, Disturbances in lipid metabolism in diabetic pregnancy - are these the cause of the problem? Best Pract. Res. Clin. Endocrinol. Metabol. 24 (4) (2010) 515-525.

[4] E. Koukkou, G.F. Watts, C. Lowy, Serum lipid, lipoprotein and apolipoprotein changes in gestational diabetes mellitus: a cross-sectional and prospective study, J. Clin. Pathol. 49 (8) (1996) 634-637.

[5] D.R. Hollingsworth, S.M. Grundy, Pregnancy-associated hypertriglyceridemia in normal and diabetic women. Differences in insulin-dependent, non-insulindependent, and gestational diabetes, Diabetes 31 (12) (1982) 1092-1097.

[6] C. Marseille-Tremblay, M. Ethier-Chiasson, J.C. Forest, Y. Giguere, A. Masse C. Mounier, et al., Impact of maternal circulating cholesterol and gestational diabetes mellitus on lipid metabolism in human term placenta, Mol. Reprod. Dev. 75 (6) (2008) 1054-1062.

[7] H.E. Miettinen, K. Rono, S. Koivusalo, B. Stach-Lempinen, M. Poyhonen-Alho J.G. Eriksson, et al., Elevated serum squalene and cholesterol synthesis markers in pregnant obese women with gestational diabetes mellitus, J. Lipid Res. 55 (12) (2014) 2644-2654.

[8] T.A. Miettinen, H. Gylling, M.J. Nissinen, The role of serum non-cholesterol sterols as surrogate markers of absolute cholesterol synthesis and absorption, Nutr. Metabol. Cardiovasc. Dis. 21 (10) (2011) 765-769.

[9] M.D. Kilby, R.H. Neary, M.I. Mackness, P.N. Durrington, Fetal and maternal lipoprotein metabolism in human pregnancy complicated by type I diabetes mellitus, J. Clin. Endocrinol. Metabol. 83 (5) (1998) 1736-1741.

[10] S.N. Pantelakis, A.H. Cameron, S. Davidson, P.M. Dunn, A.S. Fosbrooke, J.K. Lloyd, et al., The diabetic pregnancy. A study of serum lipids in maternal and umbilical cord blood and of the uterine and placental vasculature, Arch. Dis. Child. 39 (1964) 334-341.

[11] H. Merzouk, S. Madani, N. Korso, M. Bouchenak, J. Prost, J. Belleville, Maternal and fetal serum lipid and lipoprotein concentrations and compositions in type 1 diabetic pregnancy: relationship with maternal glycemic control, J. Lab. Clin. Med. 136 (6) (2000) 441-448.

[12] K. Rono, B. Stach-Lempinen, M.M. Klemetti, R.J. Kaaja, M. Poyhonen-Alho J.G. Eriksson, et al., Prevention of gestational diabetes through lifestyle intervention: study design and methods of a Finnish randomized controlled multicenter trial (RADIEL), BMC Pregnancy Childbirth 14 (2014) 70.

[13] Association AD, Diagnosis and classification of diabetes mellitus, Diabetes Care 31 (Suppl 1) (2008) S55-S60.

[14] J. Pihkala, T. Hakala, P. Voutilainen, K. Raivio, Characteristic of recent fetal growth curves in Finland, Duodecim 105 (18) (1989) 1540-1546.

[15] T.A. Miettinen, Cholesterol metabolism during ketoconazole treatment in man, J. Lipid Res. 29 (1) (1988) 43-51.

[16] T.A. Miettinen, R.S. Tilvis, Y.A. Kesaniemi, Serum plant sterols and cholesterol precursors reflect cholesterol absorption and synthesis in volunteers of a randomly selected male population, Am. J. Epidemiol. 131 (1) (1990) 20-31.

[17] A. Correani, S. Visentin, E. Cosmi, E. Ponchia, S. D'Aronco, M. Simonato, et al., The maternal-fetal gradient of free and esterified phytosterols at the time of delivery in humans, Clin. Nutr. (Edinb.) (2017). https://doi.org/10.1016/j.clnu. 2017.10.004.

[18] A.F. Vuorio, T.A. Miettinen, H. Turtola, H. Oksanen, H. Gylling, Cholesterol metabolism in normal and heterozygous familial hypercholesterolemic newborns, J. Lab. Clin. Med. 140 (1) (2002) 35-42.

[19] R. Fumagalli, R. Paoletti, The identification and significance of desmosterol in the developing human and animal brain, Life Sciences 5 (1963) 291-295. 
[20] M. Jansen, W. Wang, D. Greco, G.C. Bellenchi, U. di Porzio, A.J. Brown, et al. What dictates the accumulation of desmosterol in the developing brain? Faseb. J. 27 (3) (2013) 865-870. Official Publication of the Federation of American Societies for Experimental Biology.

[21] R.H. Neary, M.D. Kilby, P. Kumpatula, F.L. Game, D. Bhatnagar, P.N. Durrington, et al., Fetal and maternal lipoprotein metabolism in human pregnancy, Clin. Sci. 88 (3) (1995) 311-318.

[22] S.C. Couch, E.H. Philipson, R.B. Bendel, V. Wijendran, C.J. Lammi-Keefe, Maternal and cord plasma lipid and lipoprotein concentrations in wome with and without gestational diabetes mellitus. Predictors of birth weight? J. Reprod. Med. 43 (9) (1998) 816-822.

[23] N. Bansal, J.K. Cruickshank, P. McElduff, P.N. Durrington, Cord blood lipoproteins and prenatal influences, Curr. Opin. Lipidol. 16 (4) (2005) 400-408.

[24] H.J. Johnson Jr., E.R. Simpson, B.R. Carr, P.C. MacDonald, R.C. Parker Jr., The levels of plasma cholesterol in the human fetus throughout gestation, Pediatr. Res. 16 (8) (1982) 682-683.

[25] L.A. Woollett, Where does fetal and embryonic cholesterol originate and what does it do? Annu. Rev. Nutr. 28 (2008) 97-114.

[26] R.E. Ostlund Jr. J.B. McGill, C.M. Zeng, D.F. Covey, J. Stearns, W.F. Stenson, et al. Gastrointestinal absorption and plasma kinetics of soy Delta(5)-phytosterols and phytostanols in humans, Am. J. Physiol. Endocrinol. Metab. 282 (4) (2002) E911-E916.

[27] M.E. Baardman, W.S. Kerstjens-Frederikse, R.M. Berger, M.K. Bakker, R.M. Hofstra, T. Plosch, The role of maternal-fetal cholesterol transport in early fetal life: current insights, Biol. Reprod. 88 (1) (2013) 24.

[28] J. Stefulj, U. Panzenboeck, T. Becker, B. Hirschmugl, C. Schweinzer, I. Lang, et al., Human endothelial cells of the placental barrier efficiently deliver cholesterol to the fetal circulation via ABCA1 and ABCG1, Circ. Res. 104 (5) (2009) 600-608.

[29] M. Scholler, C. Wadsack, J. Metso, A.P. Chirackal Manavalan, I. Sreckovic, C. Schweinzer, et al., Phospholipid transfer protein is differentially expressed in human arterial and venous placental endothelial cells and enhances cholesterol efflux to fetal HDL, J. Clin. Endocrinol. Metabol. 97 (7) (2012) 2466-2474. 